\title{
TIDAL EVOLUTION OF LUNAR ORBIT AND EARTH ROTATION
}

\author{
SunG-Ho NA \\ Korea Astronomy and Space Science Institute, 776 Daedeok-Daero, Yuseong-Gu, Daejon 305-348, Korea \\ E-mail : sunghona@kasi.re.kr
}

(Received December 27, 2011; Revised February 16, 2012; Accepted March 5, 2012)

\begin{abstract}
In this study, I calculate the past and future dynamical states of the Earth-Moon system by using modified Lambeck's formulae. I find that the ocean tidal effect must have been smaller in the past compared to its present amount. Even though the Moon is already in the spin-orbit synchronous rotational state, my calculation suggest that it will not be in geostationary rotational state in the next billion years or so. This is due to the associated Earth's obliquity increase and slow retardation of Earth's spin and lunar orbital angular velocities. I also attempt to calculate the precessional period of the Earth in the future. To avoid uncertainties in the time scale, the future state is described by using the Earth-Moon distance ratio as independent parameter. Effects due to solar tidal dissipation are included in all calculations.
\end{abstract}

Key words : tide — lunar orbit — Earth rotation

\section{INTRODUCTION}

One of great results from lunar laser ranging (LLR) of the last forty years is the direct confirmation of lunar recession (Dickey 1994; Shelus 2001), that has been presumed since G. H. Darwin. Lunar recession is linked with the minute slowing down of Earth's spin rotation. This interesting phenomenon was discussed in a monograph (Munk \& McDonald 1960). LLR estimate of the Earth-Moon distance increase rate is $3.8 \mathrm{~cm} /$ year, and this rate is in close agreement with estimates of the Earth's tidal deceleration from other investigations, such as satellite orbit analysis and ocean tide modeling. The present lunar orbital retarding rate was estimated as $27.4 \mathrm{arcsec} / \mathrm{cy}^{2}$ by analyzing the GEOS3 orbit (Goad 1978). Lambeck has written an extensive review of these estimates up to 1970 s with some further discussions (Lambeck 1980). A brief yet wide comparison about these estimates was reported (Cazenave 1982). Study of the lunar recession naturally leads to inquiries into the lunar origin. Before the comet impact theory, there have been three ideas for the lunar origin; 'fission', 'capture', and 'binary accretion.' A good summary of these different hypotheses is given by Woolfson (Woolfson 2000). Astronomical records of eclipses bear direct information of the Earth's deceleration and the lunar retardation for a few thousand years. Paleontological evidences reveal that both periods of the Earth spin rotation and the lunar orbital rotation were shorter in the Paleozoic and the Mesozoic era than those in present days (Rosenberg and Runcorn 1975). All these phenomena are consequences of tidal interaction between the Earth and the Moon, and a schematic illustration is given in Fig. 1.

There were studies to extrapolate the dynamical state of the Earth-Moon system back into the past billion years or into the future. Among many valuable literatures related, I refer (MacDonald 1964; Kaula 1964; Goldreich 1966; Lambeck 1980; Brosche \& Sündermann 1982; Hansen 1982; de Surgy 1997; Efroimsky 2007), and cited references therein.

The closest approach of the Moon to the Earth was determined to have occurred $1.78 \times 10^{9}$ years ago by MacDonald (1964) assuming constant tidal phase lag of $2.16^{\circ}$. He opposed Darwin's fission theory, because lunar inclination angle to the Earth's equator must have been too large to be compatible with the fission process. Kaula (1964) used a systematic trigonometric series representation in describing the lunar orbit, and

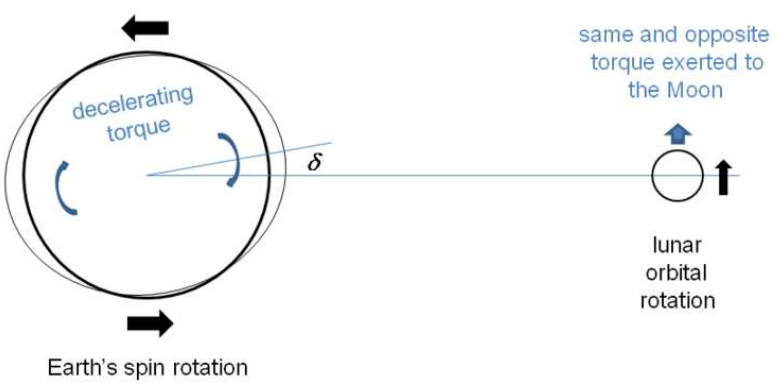

Fig. 1. - Simplified description of the Earth's deceleration by the lunar tidal torque exerted via tidal dissipation in the Earth and lunar recession due to the opposite torque, that can equivalently be ascribed to the asymmetric Earth tidal bulge. Tidal phase lag $\epsilon$ is twice the amount of angle depicted in the figure, i.e., $\epsilon=2 \delta$. 
avoided time scale problem by using tidal quality factor $\mathrm{Q}$ as a parameter. Goldreich (1966) used three different time steps in integration of his differential equations for Earth-Moon system parameters. Lambeck (1975) applied Kaula's formulation, and identified contributions of each tidal constituent in the present rate of tidal evolution. In his monograph (Lambeck 1980), Lambeck presented four chapters dealing with materials concerned with this subject. Brosche \& Sündermann (1982) edited their second monograph by compiling 19 articles, all related with this subject. Among those articles, Mignard (1982) avoided time scale problem and included diverse arguments and calculational results, and Webb (1982) composed an 'orientation averaged ocean' to represent the most generalized ocean tidal effect. Hansen (1982) reported his results on two different lunar orbital history calculations by assuming idealized paleo-ocean configurations. de Surge and Laskar (1997) calculated long term evolution of Earth's spin. They focused on the Earth's obliquity changes and its instability due to planetary perturbations. Laskar et al. (2004) extended it by including small oscillations in the Earth's obliquity superimposed on its secular increase. Efroimsky and Lainey (2007) suggested frequency dependence of the form $\omega^{0.2-0.4}$ for the body tidal Q, and they applied it for their calculation of Phobos' tidal evolution.

Many earlier studies neglected solar tidal dissipation. But solar tidal dissipation should not be ignored in long time changes of Earth's obliquity or spin angular velocity, although the angular momentum transfer from Earth's spin into Earth's orbital rotation via solar tidal dissipation cannot be easily detected. Also some of previous studies considered the $M_{2}$ tide only. Although there were academic efforts, very large uncertainty still exists in the paleo-ocean configuration and also in the amount of paleo-ocean tidal dissipation. Williams (2009) recounted that even the rough estimate of the total water amount of paleo-ocean is not available yet.

In this study, I reapproach the dynamical evolution of lunar orbit and Earth's spin rotation by using Lambeck's formulae and the following approximations; (i) an idealized frequency dependence of tidal dissipation for both body tide and ocean tide, (ii) constant solar tidal dissipation effect varying only for tidal frequency, and (iii) simple mathematical model for time dependence of ocean tidal effect.

\section{FORMULATION}

In this study, I follow Lambeck's approach (Lambeck 1975, 1980) with important modification since lunar orbital change is directly linked with change in the Earth's spin (unlike artificial satellites, which cannot alter Earth's motion). Therefore part of Lambeck's formulation was replaced with my own. Since the objective of this study is a long time history of the Earth-
Moon system over billion years, certain approximations were taken by neglecting the precession of lunar orbital plane and the small oscillatory variation of Earth's obliquity due to planetary perturbation and Earth's orbital change.

\section{$2.1 \quad$ Satellite Orbit Dynamics}

Kepler's laws of planetary motion also hold for orbital motion of satellites. The motion of satellite along its elliptical orbit in an arbitrarily oriented plane can be described by six Keplerian orbital elements $s_{i}$ ( $a, e, I, \omega, \Omega, M$; semimajor axis, eccentricity, inclination to the equator, argument of perigee, argument of ascending node, and mean anomaly). But Earth's deviation from spherical symmetry and other perturbations result in slow changes of satellite orbit. Kaula (1966) derived an expression for the time rate of Keplerian orbital elements $s_{i}$ of a satellite under perturbing potential $U$ of the form: $\frac{d s_{i}}{d t}=\sum_{j=1}^{6}\left[s_{i}, s_{j}\right]^{-1} \frac{\partial U}{\partial s_{j}}$, where $\left[s_{i}, s_{j}\right]$ is the Lagrange bracket matrix of the Keplerian elements. Among his original six equations, two following ones are relevant for this study.

$$
\begin{gathered}
\frac{d a}{d t}=\frac{2}{n a} \frac{\partial U}{\partial M} \\
\frac{d e}{d t}=\frac{1-e^{2}}{n a^{2} e} \frac{\partial U}{\partial M}-\frac{\sqrt{1-e^{2}}}{n a^{2} e} \frac{\partial U}{\partial \omega}
\end{gathered}
$$

Kaula also gave expression for gravity potential in terms of the Keplerian elements. These works of Kaula are regarded as standard treatment in the satellite orbit dynamics.

\subsection{Lambeck's Approach and Its Modification}

The force or torque exerted between the Earth and the Moon can be expressed by using formulae developed in satellite orbit dynamics. Unlike artificial satellites, the lunar mass is not negligible but about $1 / 81$ of the mass of Earth, and the present lunar orbital angular momentum is about five times larger than the present Earth's spin angular momentum. Therefore any changes in the two angular momenta should be simultaneously considered together.

Lambeck $(1975,1980)$ extended the Kaula's formulations $(1964,1966)$ to express the lunar orbital changes due to the tides on Earth raised by the Moon itself. These developments are briefly described below. First, the gravitational potential at any field point outside the Earth due to tidal deformations of the Earth can be written as the following.

$$
U(\vec{r})=\frac{G M}{a} \sum_{l=2}^{\infty} \sum_{m=0}^{l}\left(\frac{R}{r}\right)^{l+1}\left(\frac{R}{a}\right)^{l} k_{l}\left(2-\delta_{m 0}\right)
$$




$$
\begin{array}{r}
\times \frac{(l-m) !}{(l+m) !} P_{l m}(\cos \theta) \sum_{p=0}^{l} F_{l m p}(I) \\
\times \sum_{q=-\infty}^{\infty} G_{l p q}(e)\left[\begin{array}{c}
\cos \\
\text { sin }
\end{array}\right]_{l-\operatorname{modd}}^{l-m e v e n}\left(\nu_{l m p q}-m \lambda\right)
\end{array}
$$

In Eq. 3, $G$ is the constant of gravitation, $M$ is mass of tide raising body, $a$ is distance between the Earth and the body, $R$ is radius of Earth, $k_{l}$ is tidal Love number of degree $l, \vec{r}=r \hat{r}$ is field point, which is denoted by spherical coordinates - $(r, \theta, \lambda)$, and $P_{l m}(\cos \theta)$ is the Legendre function. In this study, we retain only terms up to degree $l=2$, since they provide dominant contribution (presently 98 percent). $F_{l m p}(I)$ and $G_{l p q}(e)$ are the same as defined by Kaula (1966). The position of tide raising body is expressed by the Keplerian elements, and the angle $\nu_{l m p q}$ is defined as $\nu_{l m p q}=(l-2 p) \omega+(l-2 p+q) M+m(\Omega-\theta)+\varepsilon_{l m p q}$, where $\varepsilon_{l m p q}$ is the phase delay of each tidal constituent. If the field point is expressed by another set of Keplerian elements (denoted by superscript 'sat') of a satellite, the perturbing potential for that satellite can be written as

$$
\begin{aligned}
U(\vec{r})=\frac{G M}{a} & \sum_{l=2}^{\infty} \sum_{m=0}^{l}\left(\frac{R}{a^{s a t}}\right)^{l+1}\left(\frac{R}{a}\right)^{l} k_{l}\left(2-\delta_{m 0}\right) \frac{(l-m) !}{(l+m) !} \\
& \times \sum_{u=0}^{l} F_{l m u}\left(I^{\text {sat }}\right) \sum_{\nu=-\infty}^{\infty} G_{l u \nu}\left(e^{s a t}\right) \sum_{p=0}^{l} F_{l m p}(I) \\
& \times \sum_{q=-\infty}^{\infty} G_{l p q}(e) \cos \left(\nu_{l m p q}-\nu_{l m u \nu}^{\text {sat }}+\varepsilon_{l m p q}\right)
\end{aligned}
$$

Since the Moon is affected by a perturbing force originated from the tidal deformation raised by itself, two sets of the Keplerian elements must be the same in this problem. By using Eq. 4 and the partial derivatives $\frac{d s_{i}}{d t}$ briefly described in Section 2.1, and retaining secular terms only, Lambeck $(1975,1980)$ obtained the following simple expressions for the time rates of the semi-major axis and the eccentricity of lunar orbit.

$$
\begin{aligned}
\frac{d a}{d t}= & 2 K_{l m}\left[F_{l m p}(I)\right]^{2}\left[G_{l p q}(e)\right]^{2}(l-2 p+q) \sin \varepsilon_{l m p q} \\
& \frac{d e}{d t}=K_{l m} \frac{\sqrt{1-e^{2}}}{a e}\left[F_{l m p}(I)\right]^{2}\left[G_{l p q}(e)\right]^{2} \\
\times & {\left[\sqrt{1-e^{2}}(l-2 p+q)-l+2 p\right] \sin \varepsilon_{l m p q} }
\end{aligned}
$$

where, $K_{l m}$ in Eqs. 5 and 6 is defined as follows.

$$
K_{l m}=\frac{G M_{m} k_{l}}{G\left(M_{e}+M_{m}\right) a}\left(\frac{R}{a}\right)^{2 l+1} \frac{(l-m) !}{(l+m) !}\left(2-\delta_{m 0}\right)
$$

From existing global models of ocean tide, Lambeck (1980) determined values of the equivalent phase lag

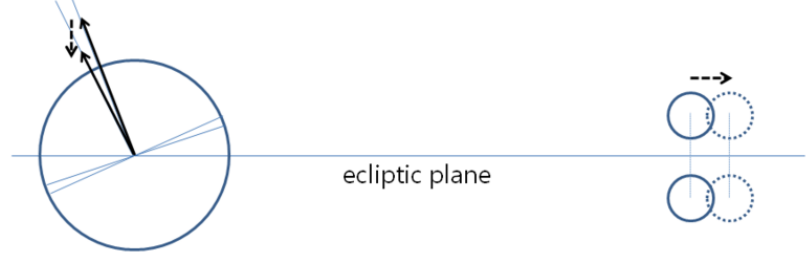

Fig. 2.- Schematic figure of the Earth-Moon system. The Earth's spin angular momentum decrease is accompanied with recession of the Moon, increase of the Earth's obliquity, and decrease of the lunar inclination to the ecliptic.

$\varepsilon_{l m p q}$. For dominant tidal constituents, his estimates of $\varepsilon_{l m p q}$ are -6.4 for $M_{2}, 6.6$ for $N_{2}, 1.7$ for $O_{1}$, and 1.8 for $K_{1}$ tides (unit: degree).

As noted earlier, the orbital angular momentum of the Moon is quite large and, in fact, plays a dominant role in the present dynamical state of the Earth-Moon system. Lunar torque exerted on the Earth results in significant amount of changes in the Earth's spin rotation over long time scale. Change in lunar orbital inclination angle to the ecliptic and change in Earth's obliquity are linked with each other via angular momentum conservation. While the Moon recedes with lunar orbital angular momentum gain, the Earth loses same amount of spin angular momentum and undergoes deceleration and obliquity increase (Fig. 2). Lambeck's formula for the change in lunar orbital inclination was

4) derived with neglecting mass and angular momentum of the Moon. Therefore, Lambeck's equation for rate of change in lunar orbital inclination should be abandoned, while his two other equations for infinitesimal changes of semimajor axis and eccentricity continue to hold.

By imposing angular momentum conservation, expressions for time rate of the Earth's obliquity and time rate of the lunar orbital inclination to the ecliptic plane are derived to be the following.

$$
\begin{gathered}
\frac{d \epsilon}{d t}=\frac{G M_{m}}{0.33 R^{2} \sqrt{G\left(M_{e}+M_{m}\right)}} \frac{\sin \epsilon}{\Omega_{e}} \\
\times \cos I\left(\frac{\sqrt{1-e^{2}}}{2 \sqrt{a}} \frac{d a}{d t}-\frac{e \sqrt{a}}{\sqrt{1-e^{2}}} \frac{d e}{d t}\right) \\
\frac{d I}{d t}=-\sin I\left(\frac{1}{2 a} \frac{d a}{d t}-\frac{e}{1-e^{2}} \frac{d e}{d t}\right)
\end{gathered}
$$

Also, expressions for the time rates of Earth's spin angular velocity and the lunar orbital angular velocity are acquired as follows.

$$
\frac{d \omega_{e}}{d t}=-\omega_{e} \cot \epsilon \frac{d \epsilon}{d t}
$$


Table 1.

The Earth-Moon system configuration parameters: their present values (upper row) ${ }^{1}$ and their present time rates (lower row $)^{2}$ used in the calculations of this study.

\begin{tabular}{cccccc}
\hline \hline $\begin{array}{c}a \\
\begin{array}{c}\text { Earth-moon } \\
\text { distance }\end{array}\end{array}$ & $\begin{array}{c}e \\
\text { lunar orbital } \\
\text { eccentricity }\end{array}$ & $\begin{array}{c}\epsilon \\
\text { Earth's } \\
\text { obliquity }\end{array}$ & $\begin{array}{c}\text { lunar orbital } \\
\text { inclination }\end{array}$ & $\begin{array}{c}\omega_{e} \\
\text { Earth's spin } \\
\text { angular velocity }\end{array}$ & $\begin{array}{c}\omega_{m} \\
\text { lunar orbital } \\
\text { angular velocity }\end{array}$ \\
\hline $3.844 \times 10^{9}$ & 0.0549 & 23.44 & 5.148 & $7.292 \times 10^{-5}$ & $2.662 \times 10^{-6}$ \\
\hline \multicolumn{7}{c}{$d a / d t$} & $d e / d t$ & $d \epsilon / d t$ & $d I / d t$ & $d \omega_{e} / d t$ & $d \omega_{m} / d t$ \\
\hline $1.198 \times 10^{-9}$ & $4.535 \times 10^{-19}$ & $2.971 \times 10^{-18}$ & $-1.375 \times 10^{-19}$ & $-5.997 \times 10^{-22}$ & $-1.244 \times 10^{-23}$ \\
\hline
\end{tabular}

${ }^{1}$ units of the parameters are $[\mathrm{m}],[$ none $],[$ degree $],[$ degree $],[\mathrm{rad} / \mathrm{s}],[\mathrm{rad} / \mathrm{s}]$.

${ }^{2}$ units of their time derivatives are $[\mathrm{m} / \mathrm{s}],\left[\mathrm{s}^{-1}\right],[\mathrm{rad} / \mathrm{s}],[\mathrm{rad} / \mathrm{s}],\left[\mathrm{rad} / \mathrm{s}^{2}\right],\left[\mathrm{rad} / \mathrm{s}^{2}\right]$.

$$
\frac{d \omega_{m}}{d t}=-\frac{3 \omega_{m}}{2 a} \frac{d a}{d t}
$$

In derivation of Eqs. 8-11, the total angular momentum component perpendicular to the ecliptic plane is claimed to be constant. This is due to the solar torque, which ceaselessly affects the lunar orbit and is responsible for the precession of the lunar orbital angular momentum component parallel to the ecliptic plane. In actual calculation, Eq. 10 was added with another term, which corresponds to the solar tidal dissipation effect.

The sets of present values of the parameters of the Earth-Moon system dynamic configuration and their time rates are given in Table 1 . These values were taken as the initial values for the calculations of this study.

\subsection{Other Consideration and Approximation}

A strong constraint on the past dynamical state of the Earth-Moon system can be acquired from fossil records. One particular estimate was adopted for the paleo-Earth's spin angular velocity at 0.5 billion years ago as $\omega_{e}=8.4 \times 10^{-5}(\mathrm{rad} / \mathrm{s})$ from Lambeck (1980). In the later sections of this study (Section 3.1 and 3.3), this value is found compatible with the paleontological evidence. Also this value matches with the present lunar recession rate $\frac{d a}{d t}=3.8 \mathrm{~cm} / \mathrm{yr}$. Based on certain paleontological records in southern Australia, Williams (2000) has reported different estimate, that is smaller by a factor of half. In this study, Wiliams' estimate of paleo-Earth angular velocity is ignored, because (i) Lambeck's estimate is in good match with the lunar recession rate, and (ii) Williams obtained his estimate from only a restricted area.
Calculation using the values for the equivalent phase angles $\varepsilon_{l m p q}$ suggested by Lambeck (1980) led to results that were incompatible with paleontological evidences. Using one fourth of the values suggested by him found to lead to correct state of the Earth-Moon system in the Paleozoic and the Mesozoic era.

Tidal phase lag has certain frequency dependence, although its functional character is not well known. Following Efroimsky and Lainey (2007), a simple relation was chosen as follows.

$$
\varepsilon_{l m p q}=\varepsilon_{l m p q}^{0}\left(\frac{\omega_{e}^{0}}{\omega_{e}}\right)^{0.3}
$$

Uncertainty exists for the true dynamical evolution of the Earth-Moon system in the geological past. Lack of knowledge of the paleo-ocean configuration is one such difficulty. Later in this study (Section 3.3-3.4), body tide and ocean tide are separated in considering their past amount of dissipation effect. And a simple power law relation for the paleo-ocean tidal effect is tested. In this study, for simplicity we consider only four main constituents - $M_{2}, N_{2}, O_{1}$, and $K_{1}$ of the lunar tide. According to Lambeck (1980), these four constituents play the major role in the lunar tidal evolution of the Earth-Moon system: 95 percent in the Earth-Moon distance evolution and 84 percent in the lunar orbital eccentricity evolution.

\section{CALCULATION AND RESULT}

\subsection{Past State Calculation with Constant Tidal Parameters}

By assigning values for the present equivalent phase angles as suggested by Lambeck and its frequency dependence as in Eq. 12 for the past state calculation, it was found that the Moon should have been at close 

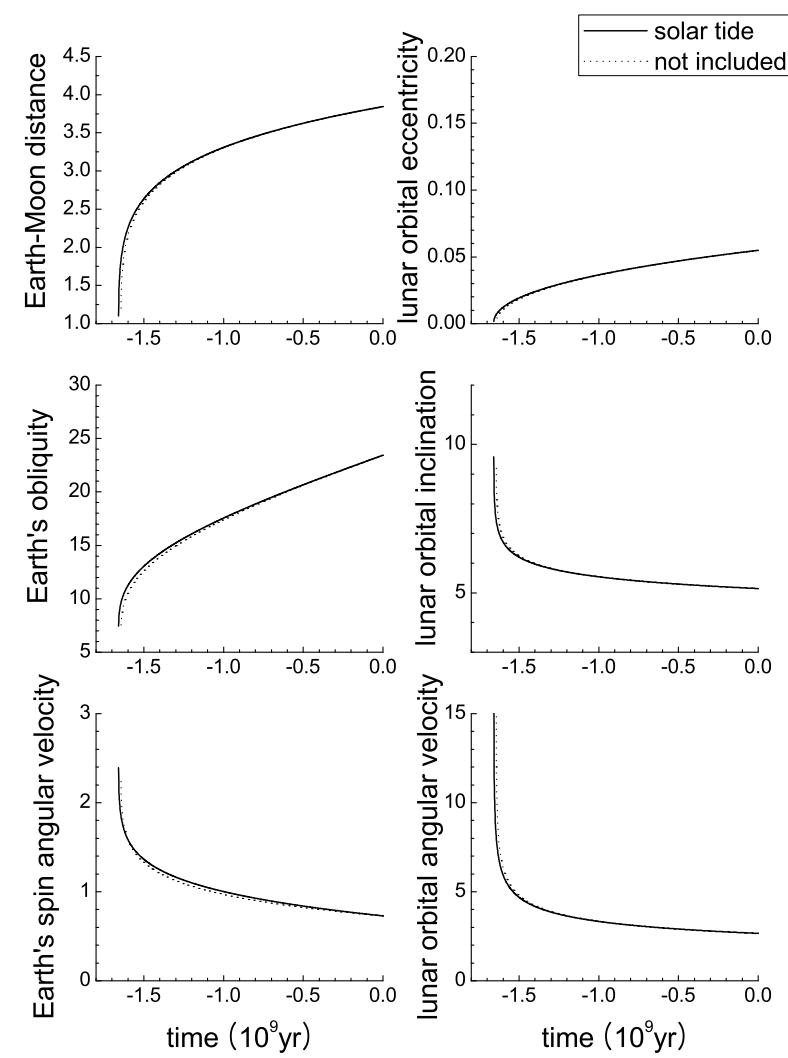

Fig. 3. - The past Earth-Moon system configuration calculated by the modified Lambeck's approach of this study. Solid lines correspond to those including solar tidal dissipation, while dotted lines correspond to those not including it. The units are $\left[10^{8} \mathrm{~m}\right]$, [none], [deg], [deg], $\left[10^{-4} \mathrm{rad} / \mathrm{s}\right]$, and $\left[10^{-6} \mathrm{rad} / \mathrm{s}\right]$ from left top.

approach to the Earth within past 0.4 billion years. By considering $M_{2}$ tide only, it was also found that similar situation should have existed within past 0.44 billion years. Moreover these calculations resulted in large differences from paleontological records of the Paleozoic and the Mesozoic era. Therefore, the equivalent phase angles were all reduced by a factor of one fourth, and the subsequent results agreed with the paleo-Earth's rotation of $\omega_{e}=8.4 \times 10^{-5}(\mathrm{rad} / \mathrm{s})$ at 0.5 billion years ago. The result of these calculations are shown in Fig. 3.

Although the paleo-Earth rotation condition is fulfilled, the calculation as shown in Fig. 3 indicated close lunar approach at 1.66 billion years ago. It was also found that at the paleozoic era, the lunar orbital eccentricity $e$ decreased to zero, the Earth's obliquity $\epsilon$ decreased to 7 degrees, the lunar orbital inclination $I$ increased to 10 degrees, the Earth's spin angular velocity $\omega_{e}$ increased over three times of its present value,

and the lunar orbital angular velocity $\omega_{m}$ increased over five times of its present value, at that state. Thus, according to this calculation, Moon was in the beginning stage of dynamical evolution at around 1.66 billion years ago. Knowing that the lunar age is over 4 billion years, this result should lead to 'capture hypothesis' for the lunar origin, which is quite unlikely and not generally accepted.

\subsection{Future State Calculation with Constant Tidal Parameter}

The future dynamical state of the Earth-Moon system from the present into the future of 5 billion years was determined. All the calculation schemes were the same as in Section 3.1, except that the time flow direction was reversed. The result is illustrated in Fig. 4. Although the state in the near future will turn out to be as described in Fig. 4, the prediction of the dynamical state of the Earth-Moon system is uncertain due
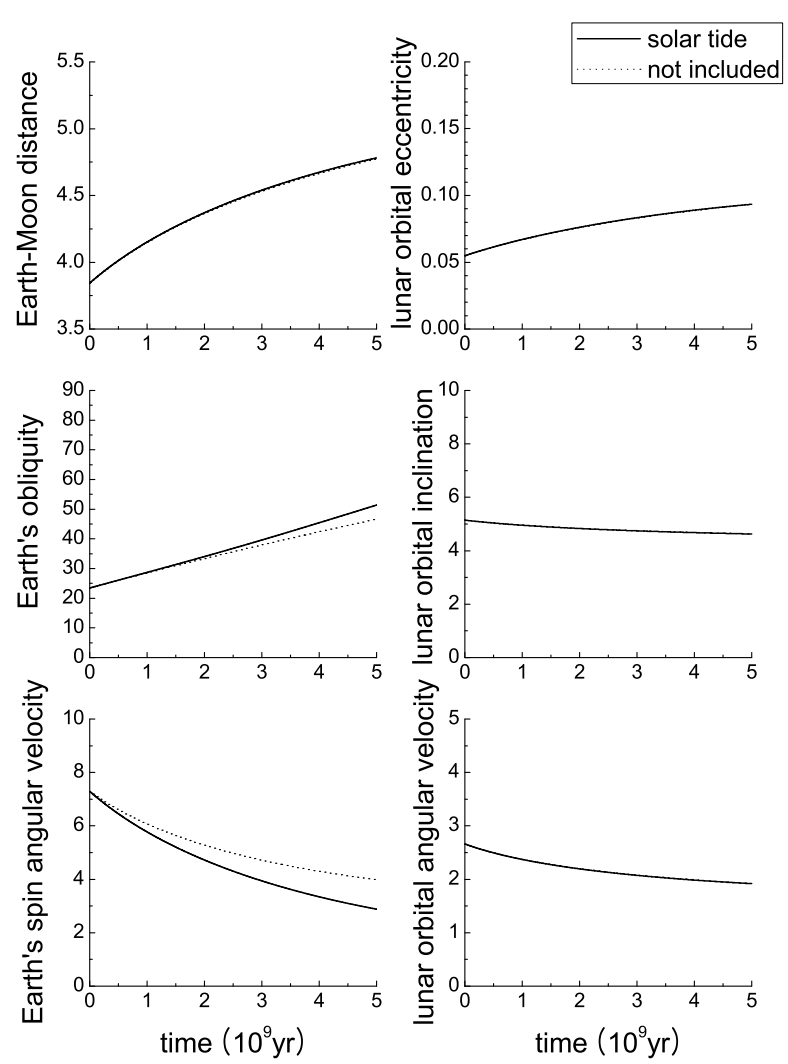

Fig. 4.- The future 5 billion years Earth-Moon system configuration calculated by the modified Lambeck's approach of this study. Solid lines correspond to those including solar tidal dissipation, while dotted lines correspond to those not including it. The units are $\left[10^{8} \mathrm{~m}\right]$, [none], [deg], $[\mathrm{deg}],\left[10^{-5} \mathrm{rad} / \mathrm{s}\right]$, and $\left[10^{-6} \mathrm{rad} / \mathrm{s}\right]$ from left top. 
mainly to unknown future ocean configuration.

Calculations including solar tidal dissipation to the future 5 billion years indicate gradual increase of the Earth-Moon distance $a$ up to $480,000 \mathrm{~km}$ and the lunar orbital eccentricity $e$ increase to 0.094. Further, the Earth's obliquity $\epsilon$ increase to $51^{\circ}$, the lunar orbital inclination $I$ decrease to $4.6^{\circ}$, the Earth's spin angular velocity $\omega_{e}$ decrease to 40 percent of present value, and the lunar orbital angular velocity $\omega_{m}$ decrease to 72 percent of present value, at that time. If the small tidal dissipation inside of Moon due to lunar libration was included into calculation, the estimate of lunar orbital eccentricity $e$ would be slightly less than the one shown in Fig. 5. It is found that four parameters $a, e, I$, and $\omega_{m}$ of the future state are not significantly affected by solar tidal dissipation. But several percent increase of the Earth's obliquity $\epsilon$ and more than ten percent reduction of the Earth's spin angular velocity $\omega_{e}$ are predicted in future 5 billion years.

If lunar orbital evolution is approximated by neglecting Earth obliquity and lunar inclination to the ecliptic, geostationary Moon could be found in a far future. A calculation predicts this synchronous period as 48 days and corresponding Earth-Moon distance as 1.46 times of its present value. But, as seen in Fig. 4, due to the increase of Earth obliquity, tidal energy dissipation will not efficiently induce angular momentum transfer to lunar orbital motion in the future, and it is unlikely that the Moon will be in geostationary state.

It is noted here that the accuracy of my calculation of the dynamical state of the Earth-Moon system in the future 5 billion years is uncertain and contingent upon the consistency of tidal dissipation in the Earth during the future times. If changes occur in the tidal dissipation rate of the Earth-Moon system, the future state will consequently differ from the calculational result as shown in Fig. 4. While one cannot predict the exact future tidal dissipation rate, dynamical state of the Earth- Moon system can be traced by using the Earth-Moon distance as the independent variable. In a later section, the future state of the Earth-Moon system is obtained by using a scale factor of Earth-Moon distance to avoid the problem of dissipation rate uncertainty and time scale.

\subsection{Considering of Variable Paleo-Ocean Tide - Simple Power Law Approximation}

Tidal effect in the ocean forms the dominant part of tidal dissipation in the Earth and the dynamical evolution of the Earth-Moon system. Assuming a constant tidal phase lag during the whole geological past, it was found that the Moon should have been located near the Roche limit around 1.7 billion years ago (see Section 3.1). Since the Moon was formed 4.5 billion years ago, the discrepancy between the two - 1.7 and 4.5 billion years cannot be explained. Considering that the paleo-ocean configuration is mostly unknown (Williams 2009), one could proceed by reducing the ocean tidal

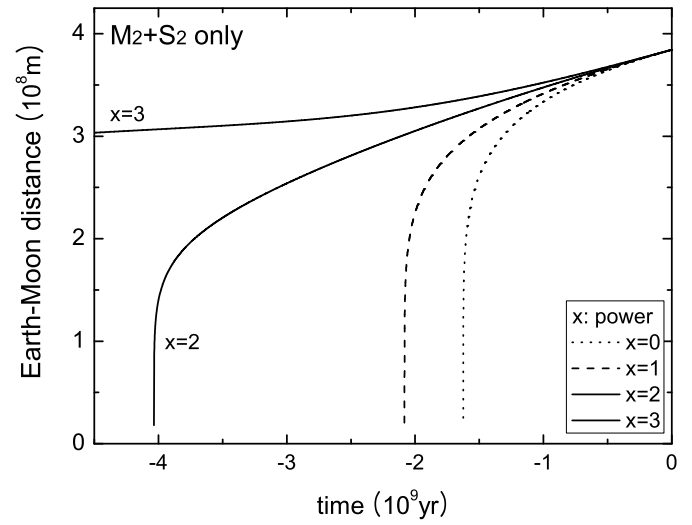

Fig. 5. - Calculated Earth-Moon distance with different power assigned for ocean tidal effect. $M_{2}$ and $S_{2}$ constituents only are considered.

contribution in the past in order to investigate the possibility of Moon's closest approach happening at around 4.5 billion years ago. After Egbert and Ray (2001), the body tide contribution was assumed to be 5 percent of the present total amount of tidal dissipation and constant throughout the whole geological past. In fact, this estimate of 5 percent has been confirmed as 5.5 percent by Mathews and Lambert (2009). A simple power law for the time dependence of the ocean tidal effect was assumed, so that Eq. 12 is replaced by the following relation Eqs. 13-14

$$
\begin{gathered}
\varepsilon_{l m p q}=\left[\varepsilon_{l m p q}^{\text {body }}+\varepsilon_{l m p q}^{\text {ocean }}\right]\left(\frac{\omega_{e}^{0}}{\omega_{e}}\right)^{0.3} \\
\varepsilon_{l m p q}^{\text {ocean }}(t)=\varepsilon_{l m p q}^{\text {ocean }}\left(t_{0}\right)\left(\frac{t}{t_{0}}\right)^{X}
\end{gathered}
$$

Based on Eqs. 13-14, calculation for the dynamical state of the Earth-Moon system in the past was again performed with different values for the power $X$. First, only $M_{2}$ tide and $S_{2}$ tide were considered. In fact, the solar tidal dissipation effect is directly inserted to Eq. 10. The results are shown in Fig. 5, where only the change of the Earth-Moon distance is illustrated.

As shown in Fig. 5, the Moon is supposed to be at its close approach to the Earth at 2.1 billion years ago by assuming the ocean tidal effect had varied linearly in time. The same situation is found at about 4 billion years ago with quadratic ocean tidal effect. As noted above, exact amount of the ocean tidal effect in the geological past is not known. Still the calculations demonstrate that the Moon could have been at its beginning stage of its dynamical evolution at 4.5 billion years ago, provided that the paleo-ocean tidal effect was somehow smaller than its present amount. 


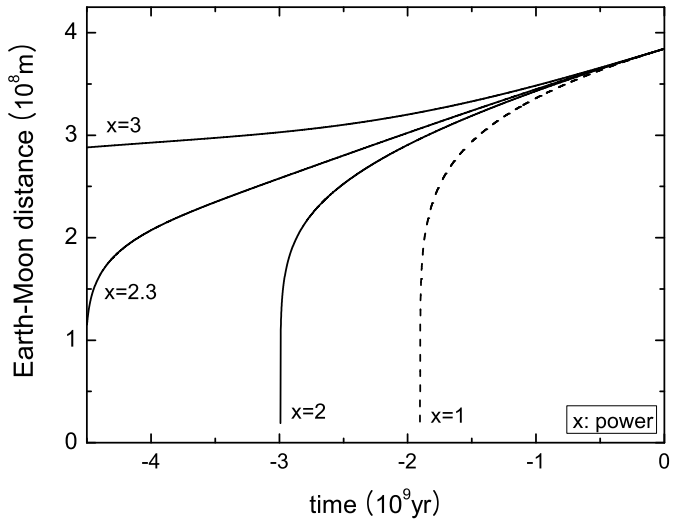

Fig. 6. - Calculated Earth-Moon distance with different power assigned for ocean tidal effect. $M_{2}, S_{2}, N_{2}, O_{1}$, and $K_{1}$ constituents are considered.

Retaining five constituents - $M_{2}, S_{2}, N_{2}, O_{1}$, and $K_{1}$, in the ocean tidal dissipation, the calculation was again repeated for different values for the power $X$. The calculated Earth-Moon distance curves are shown in Fig. 6. Compared with the former result, there are gradual shifts along time axis for the same power assigned, which is readily conceivable. It is found that two distinct approaches of ' $M_{2}$ plus idealized solar tidal dissipation' and 'five tidal constituents' yield results comparable to each other. In other words, ' $M_{2}$ and $S_{2}$ ' do the dominant role in this phenomena.

\subsection{Precession of the Earth in Future}

Based on the calculation in Section 3.2, future precession of the Earth is considered. Earth's precessional period $T^{P R E C}$ is $2 \pi / \Omega^{P R E C}$, where precessional angular velocity $\Omega^{P R E C}$ is given by the following (Danby 1992).

$$
\Omega^{P R E C}=\frac{3 G}{2 \omega_{e}} \frac{C-A}{C}\left(\frac{M_{m}}{a^{3}}+\frac{M_{\odot}}{r^{3}}\right) \cos \epsilon
$$

In Eq. 15, $C$ and $A$ are two principal moments of inertia of the Earth, $r$ is the radius of Earth orbital rotation, i.e., is presently 1 A.U., $M_{\odot}$ is the solar mass, and others are the same as defined before. The two terms of $\Omega^{P R E C}$ can be denoted as $\Omega_{\text {lunar }}^{P R E C}$ and $\Omega_{\text {solar }}^{P R E C}$, where the subscripts lunar and solar denote the precessional torques exerted by the Moon and the Sun respectively. Calculated periods of precession $T^{P R E C}$ of two cases, one for the five tidal constituents (case 1) and the other for $M_{2}$ and $S_{2}$ constituents only (case 2) are drawn in Fig. 7 (top). Also in the same figure, separate precessional torques are drawn. It is found that the period of Earth's precession increases with time, from present value of 25,800 years to about its twice amount in 5 billion years. de Surgy and Laskar (1997) predicted instability of Earth's spin rotation, when the Earth's obliquity angle becomes larger than $60^{\circ}$. The following arguments are based on the calculation in Section 3.2. Assuming no solar tidal dissipation and same ocean tidal dissipation in the future, Laskar's instability would not occur even in 5 billions years. However, the Earth's spin angular velocity in future 4 billion years will be reduced by additional 10 percent due to solar tidal dissipation. Therefore, the Laskar's instability may occur after 4 billion years. To avoid time scale uncertainty, one may state that Laskar's instability will not occur until Earth-Moon distance will exceed about 1.2 times of its present amount. It should be noted that coremantle friction is neglected in this study.

\section{DISCUSSION}

Lambeck's approach has been proven to be efficient, in this study, to calculate the dynamical history of the Earth-Moon system, provided with the correction of his third formula for time rate of lunar inclination, $d I / d t$ as in Section 2.2. One fourth of each tidal phase angle of Lambeck's estimate is found compatible to the present lunar recession rate of $3.8 \mathrm{~cm} / \mathrm{yr}$ and also to paleontological evidences.

From the result obtained in Sections 3.1 and 3.3, one can contemplate the origin of the Moon. First, large
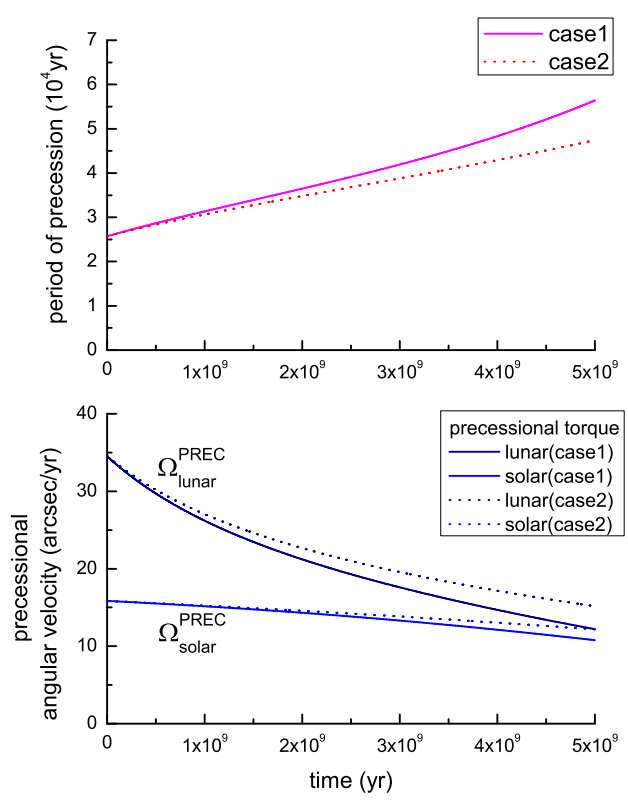

Fig. 7.- Calculated period of precession and precessional angular velocity. First, all lunar tidal constituents and $S_{2}$ constituent are considered - case1. Also only $M_{2}$ and $S_{2}$ are considered - case2. Two kinds of precessional torque contributions from the Moon and the Sun are separately shown in the lower panel. 
value of lunar orbital inclination at the beginning stage of Earth-Moon tidal evolution puts 'fission hypothesis' - Moon was originated by fission due to rotational instability of early Earth - as unrealistic. 'Binary accretion hypothesis' is also regarded as unrealistic on the same ground. Gerstenkorn event, which assumed 'capture of the Moon,' has not been favored generally. 'Capture hypothesis' is discarded in this study, because its scenario - large angular momentum transfer to the approaching Moon in a retrograde orbit by extreme tidal dissipation within a few days should demand coincidence of too small probability. 'Impact hypothesis' remains to be the only acceptable theory. However, without reducing the tidal dissipation in the PreCambrian eon, one should assume the event of impact and lunar formation at only 1.7 billion years ago. Since the lunar age is known to be comparable to that of Earth, reduction of ocean tidal dissipation as in Eqs. 13-14 or other substitute is necessary. There is no supporting evidence for the simple power law of Eq. 14. But without enough information of paleo-ocean configuration and its water amount, it is impossible to correctly estimate the ocean tidal dissipation in the past.

From the similarity of the two different sets of calculations as shown in Fig. 5 and Fig. 6, it was found that $M_{2}$ and $S_{2}$ constituents together well approximate elaborate approach with more terms and other additional considerations.

As noted before, future state calculation also has uncertainty problem on the amount of ocean tidal dissipation. But future ocean configuration change would be relatively slower, because the plate tectonic movement is slowing down. The amount of ocean water in the future is also unknown. With such limited information, first conjecture might be to take a constant value for the tidal phase lag angle in Eq. 13. Section 3.2 was based on this argument.

In both the past and future state calculation, it is possible to avoid time scale problem and to take the Earth-Moon distance as the fundamental variable rather than time. There were such kind of studies, for example, (Goldreich 1966; Mignard 1982). Advantage of these studies is that true tidal dissipation rate is not necessarily known. Exact validity of the Earth-Moon distance as the fundamental variable is feasible, when solar tidal dissipation does not exist and relation between tidal torque and tidal dissipation is quantified through time steps. Nevertheless, such change of the variables might be the only systematic way to avoid tidal dissipation rate uncertainty.

The former result shown in Fig. 4 in Section 3.2 can be alternatively expressed by defining a scale factor $\tau=a / a_{0}$ as the fundamental variable, where $a_{0}$ is the present Earth-Moon distance. Calculation was halted when Earth's obliquity exceeds 80 degrees. The result is shown in Fig. 8. In fact, about twice and thrice longer future time spans were needed for Fig. 8. Other calculational result, such as future precessional period,
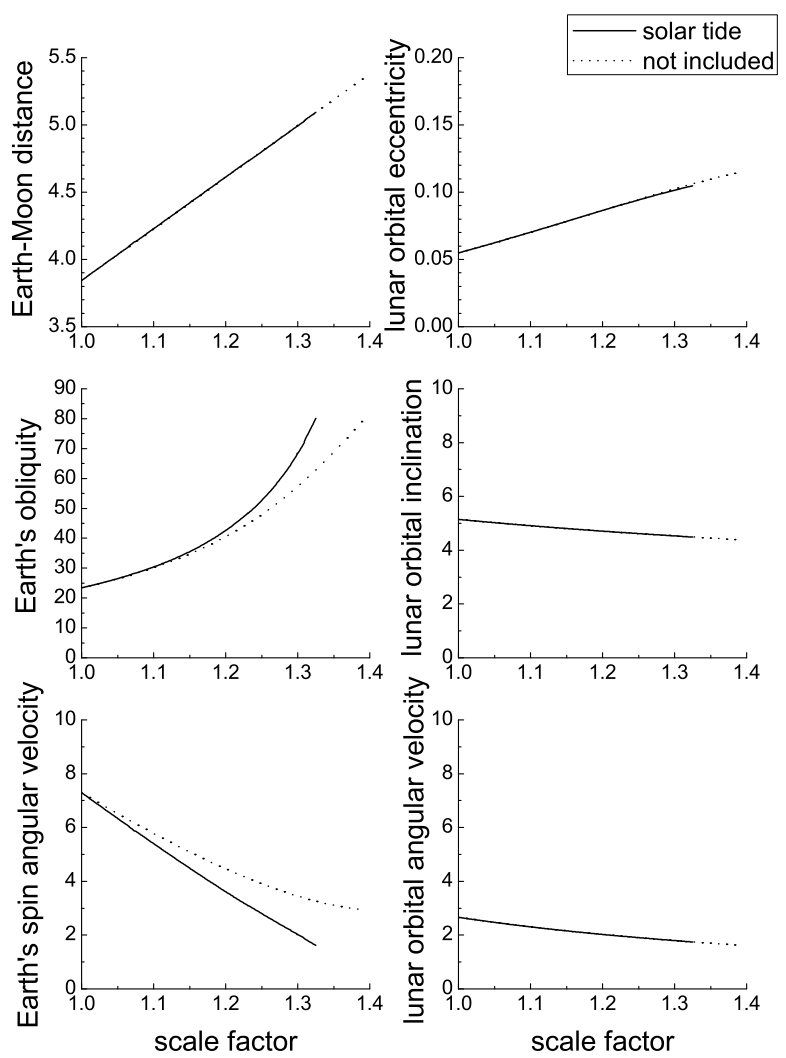

Fig. 8.- The future Earth-Moon system configuration calculated by the modified Lambeck's approach of this study. Horizontal axes is now the scale factor $\tau$ rather than time. Solid lines correspond to those including solar tidal dissipation, while dotted lines correspond to those not including it. The units are $\left[10^{8} \mathrm{~m}\right]$, [none], [deg], [deg], $\left[10^{-5} \mathrm{rad} / \mathrm{s}\right]$, and $\left[10^{-6} \mathrm{rad} / \mathrm{s}\right]$ from left top.

can be re-scaled in the same way by using the scale factor $\tau$. As noted above, this kind of transformation also is not rigorously valid, but an approximate one.

\section{SUMMARY}

Extrapolation of the dynamical state of the EarthMoon system into the past/future billion years has been attempted by using the Lambeck's formulation with correction. According to the calculation with assumption of constant tidal dissipation throughout the geological past, the Moon should be placed near its Roche limit at 1.66 billion years ago. By reducing ocean tidal effect in the past, it was found that such close approach could have happened at much earlier time, even 4.5 billion years ago. Therefore, past ocean tidal effect is believed to have been smaller in the past. Comparable results have been attained for the calcu- 
lations with $M_{2}$ tide only. All the calculations in this study were designed to be compatible with the paleontological evidence as well as the present lunar recession rate. Unlike simplified calculation for planar geometry of Earth-Moon system, it was found that the Earth spin will not slow down enough to be synchronous rotation with the lunar orbital motion even in billion years future. Rather Earth's obliquity will increase in great amount, while Earth's spin retardation occur slowly. Also Earth's obliquity instability due to the planetary perturbation will not occur until the Earth-Moon distance increase by 20 percent, which may happen after 4 billion years or so.

\section{ACKNOWLEDGMENTS}

This work was supported by the Korea Astronomy and Space Science Institute through the SLR system development program for space geodesy funded by the Ministry of Education, Science, and Technology (MEST). The author is grateful to his former supervisor Prof. Wooil Moon for kind introduction of this topic years ago. Kind suggestions from an anonymous reviewer were helpful to enhance this article. Finally, every pre-existing work as cited is appreciated.

\section{REFERENCES}

Brosche, P., \& Sündermann, J. 1982, Tidal Friction and the Earth's Rotation II (ed) Springer-verlag, Berlin Heidelberg

Cazenave, A. 1982, Tidal Friction Parameters from Satellite Observations, in Tidal Friction and the Earth's Rotation II ed. by Brosche, P., \& Sündermann, J., Springer-verlag, Berlin Heidelberg, 4

Danby, J. M. A. 1992, Fundamentals of Celestial Mechanics 2nd ed., Willman-Bell, Inc., Ch. 13

Dickey, J. O. 1994, Lunar Laser Ranging: A Continuing Legacy of the Apollo Program, Science, 265, 482

Efroimsky, M., \& Lainey, V. 2007, Physics of bodily tides in terrestrial planets and the appropriate scales of dynamical evolution, J. Geophys., Res. 122, E12033, doi:10.1029/2007JE002908

Egbert, G. D., \& Ray, R. D. 2001, Estimates of $\mathrm{M}_{2}$ Tidal Energy Dissipation from TOPEX/Poseidon Altimeter Data, J. Geophys., Res. 106, C10, 22475

Goad., C. C., \& Douglas, B. C. 1978, Lunar Tidal Accerleration Obtained From Satellite-Derived Ocean Tide Parameters, J. Geophys., Res. 83, B5, 2306

Goldreich, P. 1966, History of the Lunar Orbit, Rev. Geophys., 4, 411

Hansen, K. 1982, Secular Effects of Oceanic Tidal Dissipation on the Moon's Orbit and the Earth's Rotation, Rev. Geophys., Sp. Phys., 20, 457

Kaula, M. 1964, Tidal Dissipation by Solid Friction and the Resulting Orbital Evolution, Rev. Geophys., 2, 661
Kaula, M. 1966, Theory of Satellite Geodesy, Blaisdell Pub. Co., Ch. 3

Lambeck, K. 1975, Effects of Tidal Dissipation in the Oceans on the Moon's Orbit and the Earth's Rotation, J. Geophys., Res. 80, 2917

Lambeck, K. 1980, The Earth's Variable Rotation: Geophysical Causes and Consequences, Cambridge Univ. Press, Cambridge, Ch. 5-6, 10-11

Laskar, J., Robutel, P., Joutel, F., Gastineau, M., Correia, A. C. M., \& Levrard, B. 2004, A Long-Term Numerical Solution for the Insolation Quantities of the Earth, A\&A, 428, 261

MacDonald, G. J. F. 1964, Tidal Friction, Rev. Geophys., 2, 467

Mathews, P. M., \& Lamber, S. B. 2009, Effect of Mantle and Ocean Tides on the Earth's Rotation Rate, A\&A, 493, 325

Mignard, F. 1982, Long Time Integration of the Moon's Orbit, in Tidal Friction and the Earth's Rotation II ed. by Brosche, P., \& Sündermann, J., Springerverlag, Berlin Heidelberg, 67

Munk, W. H., \& MacDonald, G. J. F. 1960, The Rotation of the Earth, Cambridge Univ. Press., Cambridge, Ch. 11

Rosenberg, G. D., \& Runcorn, S. K. 1975, Growth Rythms and the History of the Earth's Rotation (ed), Wiley, London \& New York

Shelus, P. J. 2001, Lunar Laser Ranging: Glorious Past and a Bright Future, Sur. Geophys., 22, 517

de Surgy, O. N., \& Laskar, J. 1997, On the Long Term Evolution of the Spin of the Earth, A\&A, 318, 975

Webb, D. J. 1982, On the Reduction in Tidal Dissipation Produced by Increases in the Earth's Rotation Rate and Its Effect on the Long-Term History of the Moon's Orbit, in Tidal Friction and the Earth's Rotation II ed. by Brosche, P., \& Sündermann, J., Springer-verlag, Berlin Heidelberg, 210

Williams, G. 2000, Geological Constraints on the PreCambrian History of Earth's Rotation and the Moon's Orbit, Rev. Geophys., 38, 37

Williams, G. 2009, Water, the Solid Earth, and the Atmosphere: The Genesis and Effects of a Wet Surface on a Mostly Dry Planet in Treatise on Geophysics v. 9, Evolution of the Earth, ed. by Stevenson, D., Ch. 5

Woolfson, M. M. 2000, The Origin and Evolution of the Solar System, IOP Pub., Bristol \& Philadelphia 